\title{
Vignettes
}

\section{Understanding the Maternal Experience of Opioid Use Disorder through Qualitative Storytelling: Innovative Undergraduate Research}

Heide S. Temples, Mary Ellen Wright, Olivia Chafe, Haley McKee, Emily Guthrie

Clemson University, heidet@g.clemson.edu

\section{doi: $10.18833 /$ spur/4/2/9}

Large social problems necessitate multidisciplinary solutions from a variety of research methodologies. The project's focus was to engage preprofessional health students using qualitative research and self-reflection to address the complex issues surrounding the underserved population of mothers with substance use disorder. The students reported development in empathy, understanding a complex social problem, and creating innovative solutions to improve health-care services.

The Creative Inquiry (CI) Program at Clemson University is an undergraduate research program with a teambased investigation led by faculty mentors to provide student research experience and course credit. Students reviewed and selected topics from descriptions of research opportunities on the CI website and were interviewed and selected. This innovative qualitative study aimed to understand the maternal experience during pregnancy and parenting while battling Opioid Use Disorder (OUD) in a rural southeastern region of the United States. Participants were recruited by collaborating with local, nonprofit, government-supported recovery centers. The Institutional Review Board of Clemson University in Clemson, SC, approved this study (IRB Exempt Protocol IRB2018-317).

Each student's preparation included reading and providing written personal reflections on two books. Dreamland (Quinones 2016) provided an overview of the etiology of the opioid crisis in the United States. A Street Cat Named Bob (Bowen 2012) told an autobiographical account of the challenges of recovering from heroin. This book served to connect students with addiction issues and to humanize people who struggle with addiction to further develop a sense of empathy.

The students and faculty collected 11 stories with a dedicated phone, only used for mothers calling to anonymously share their stories of addiction. After verbal consent to record the conversation and voluntarily share her story, the phone number of the incoming call was deleted. The qualitative storytelling narrative used open-ended questions to allow participants to share what they considered valuable in their story rather than have researchers ask predetermined questions, which could have influenced answers. After the interview ended, student researchers entered their thoughts in a journal, and faculty made themselves available to listen if undergraduates struggled with their emotions. Two students transcribed each story, removing any identifying information other than the assigned participant code and deleting the audio recording to prevent voice recognition of the participant. The data that remains for analysis is a coded word document without names or details to prevent identifying the storyteller. Maternal interviews were analyzed initially by the students for underlying themes using the Atlas.ti program, and the faculty members performed the final thematic analysis and validation.

A double-entry reflective journal of their interviews and readings allowed students to reflect, share, and discuss ideas and questions within the social context of substance use and the opioid epidemic at monthly seminars. The students also were required to complete a take-home quiz on the books' contents. Students learned and applied qualitative research methods based on theoretical frameworks relative to the research project, including Nursing as Caring by Boykin and Schoenhofer (2001) and the Ecological Social System Theory by Bronfenbrenner (1986). The students applied the theme of positive or negative social support while using Bronfenbrenner's system to further understand the complex social problem of OUD.

This research and collaboration model resulted in the dissemination of 11 posters and 2 podium presentations at 7 international, national, regional, and local professional health-care conferences supported by four competitively awarded travel grants. The dissemination of the research at nursing conferences allowed health professionals to better support families with OUD and improve health-care services for patients. Most undergraduate participants in this project were recently accepted to nursing, medical, or pharmacology programs. Understanding the social issues surrounding patient care will be advantageous for these future health-care professionals.

The publication of a book (Wright and Temples 2019) and three submitted manuscripts are products of the pedagogical teaching. The book was written to promote further understanding of the experiences of mothers with addiction and recovery and to encourage society to positively support this at-risk population. Additionally, most of the royalty funds generated are returned to assist future research on this topic through the university's research 
foundation. The research team plans to send copies of the book and informative letters to stakeholders in public, political, health, and religious organizations to spread awareness for an often overlooked vulnerable population.

This project is part of the university's Center for Research on Health Disparities, which focuses on comprehensive, culturally sensitive, and community-based research that improves health outcomes and enhances quality of life. The center links faculty, students, and community members to advance research of the complex causes of health disparities. Applying a nontraditional research model and partnering with the nonprofit recovery center industry helped to prepare preprofessional health students to understand the complexity of the issues.

\section{References}

Bowen, James. 2012. A Street Cat Named Bob: And How He Saved My Life. New York: St. Martin's Press.

Boykin, Anne, and Savina O. Schoenhofer. 2001. Nursing as Caring: A Model for Transforming Practice. Boston: Jones and Bartlett.

Bronfenbrenner, Urie. 1986. Ecology of the Family as a Context for Human Development: Research Perspectives. Developmental Psychology 22: 723-742. doi: 10.1037/0012-1649.22.6.723

Quinones, Sam. 2016. Dreamland: The True Tale of America's Opiate Epidemic. New York: Bloomsbury.

Wright, Mary Ellen, and Heide S. Temples. 2019. I Do Love My Baby: Stories of Mothers with Substance Addiction and Recovery. Seattle: Amazon Kindle Direct Publishing.

\section{Undergraduate Images of Research + Arts Competi- tion: Lessons Learned and Future Directions}

Lorraine S. Wallace, Mikafui Dzotsi, and Kayla Daniel

The Ohio State University,wallace.621@osu.edu

\section{doi: $10.18833 /$ spur/4/2/11}

Universities and professional societies have launched interactive and engaging platforms for individuals to showcase their research activities (Cuff et al. 2019; Reichle 2019; University of Leicester n.d.; University of Saskatchewan n.d.). In 2018, the Office of Undergraduate Research \& Creative Inquiry (UR\&CI) at The Ohio State University (OSU) established an Images of Research + Arts (IR+A) Competition for undergraduates to capture and share the essence of their research in a unique and visually stimulating format (UR\&CI 2020). This vignette describes the process, lessons learned, and future directions of the IR+A Competition.

UR\&CI worked alongside campus partners to promote and encourage IR $+\mathrm{A}$ Competition submissions. Over a two-month period, undergraduates submitted materials via a Qualtrics survey, which included demographic and academic information, image generation details (e.g., date and location of the image), descriptors (e.g., title, written narrative) and image upload (a minimum of 300 dpi was required). UR\&CI staff assembled and electronically distributed submissions to a multidisciplinary group of staff and faculty $(n=8)$ for an initial blind review. Reviewers evaluated submissions on connections among title, image and written narrative, originality, and overall visual impact. Reviewers identified and ranked their top five submissions. Next, UR\&CI staff compiled and tabulated the submitted rankings to identify four finalists.

Finalist submissions (title, written narrative, and image) were professionally printed on foam core boards and prominently displayed during the largest multidisciplinary OSU undergraduate research forum held each year. During the registration process, each undergraduate presenter and faculty forum judge were provided with a ballot to select their favorite submission. Completed ballots were placed in a box, sorted, and tallied to identify IR+A Competition awardees (first place, second place, third place, and honorable mention). Finalist results and accompanying images, titles, and written narratives were displayed on the UR\&CI website and disseminated through social media platforms.

In 2018 and 2019, 17 students - representing 15 academic disciplines - submitted an IR+A Competition application. Applications were submitted by students pursuing degree programs ranging from science, technology, engineering, and mathematics (e.g., biology, materials science, landscape architecture) and social and behavioral sciences (e.g., economics, nutrition) to arts and humanities (e.g., dance, history). Overwhelmingly, most students (approximately 80 percent) participating in multidisciplinary OSU undergraduate research forums are enrolled in STEM programs. Although the total number of IR+A Competition submissions was relatively small in comparison to overall undergraduate research forum participation (approximately 300), the IR+A Competition drew submissions from students outside STEM-related fields and thereby highlighted undergraduate research in often underrepresented disciplines.

Although the IR+A Competition showcases research discovery through a new lens, several aspects should be considered regarding lessons learned and future directions. The initial evaluation process was limited to reviewers identifying their top submissions. The initial evaluation process should be expanded to provide constructive feedback to all submitters. Providing constructive feedback to students is important because they could use this information to revise and resubmit their work for future IR + A Competitions or other presentation opportunities. Additionally, it would be beneficial to formally assess the IR+A Competition impact on participants, reviewers, and the general university community. 